\title{
CLASSES OF SEMI-INNER-PRODUCT SPACES $\left({ }^{1}\right)$
}

\author{
BY \\ J. R. GILES
}

1. Introduction. With the aim of carrying over Hilbert space type arguments to the theory of Banach spaces, G. Lumer [8] constructed on a vector space a type of inner product with a more general axiom system than that of Hilbert space. He defined a semi-inner-product (s.i.p.) on a complex vector space $V$ as a complex function $[x, y]$ on $V \times V$ with the following properties:

1. $[x+y, z]=[x, z]+[y, z],[\lambda x, y]=\lambda[x, y]$ for all complex $\lambda$,

2. $[x, x]>0$ when $x \neq 0$,

3. $|[x, y]|^{2} \leqq[x, x][y, y]$.

A vector space with a s.i.p. is called a semi-inner-product space (s.i.p. space). An s.i.p. space is a normed vector space with norm $\|x\|=[x, x]^{1 / 2}[8$, p. 31$]$.

For Lumer the importance of an s.i.p. space is that every normed vector space can be represented as an s.i.p. space so that the theory of operators on Banach space can be penetrated by Hilbert space type arguments.

But it is obvious that the generality of the axiom system defining the s.i.p. is a serious limitation on any extensive development of a theory of s.i.p. spaces parallel to the theory of Hilbert space. Our aim is to determine what further developments can be made for classes of s.i.p. spaces which are defined by imposing further restrictions on the s.i.p.

Outline of the paper. As a preliminary we show that a homogeneity property, $[x, \lambda y]=\bar{\lambda}[x, y]$ for all complex $\lambda$, can be imposed, and all normed vector spaces can be represented as s.i.p. spaces with this property. Our main concern is to define the class of continuous s.i.p. (uniformly continuous s.i.p.) spaces by imposing the continuity property, $\mathscr{R}\{[y, x+\lambda y]\} \rightarrow \mathscr{R}\{[y, x]\}$ for all real $\lambda \rightarrow 0$ and (uniformly) for $(x, y) \in S \times S$. We define an orthogonality relation and show that in such spaces it is equivalent to an orthogonality relation as studied by R. C. James [7]. We show that the continuity restriction on the s.i.p. is equivalent to Gâteaux (uniform Fréchet) differentiability of the norm. A representation theorem for continuous linear functionals on a uniformly convex complete continuous s.i.p. space follows on the lines of the Hilbert space theorem. Defining uniform s.i.p. spaces as uniformly convex, complete, uniformly continuous s.i.p. spaces we have, as for Hilbert space, that the dual can be identified as a uniform s.i.p. space with s.i.p. defined by $\left[f_{y}, f_{x}\right]=[x, y]$.

Received by the editors December 22, 1965 and, in revised form, April 12, 1967.

(1) This paper is part of the research undertaken towards a doctoral thesis at the University of Newcastle, Australia. The author wishes to express his thanks to Professor G. Szekeres for his encouragement and guidance. 
As an example, we show that H. Rund's work [9], [10] suggests that Minkowskian spaces can be regarded as affine spaces associated with real finite-dimensional uniform s.i.p. spaces. This approach shows that the principal dual properties are independent of the differentiability of the norm as a function of real variables. As a further example, we show that the real $\mathscr{L}_{p}$-spaces $(1<p<\infty)$ can be readily established as uniform s.i.p. spaces. Hence we can deduce immediately the representation theorem for continuous linear functionals and properties of the dual.

Notation. In a normed vector space $V$ we denote the unit sphere $\{x \mid\|x\|=1\}$ by $S$; we denote the dual space by $V^{*}$ and the unit sphere in the dual space by $S^{*}$.

2. Continuous s.i.p. spaces and uniform s.i.p. spaces. We note at the outset that the imposition of a homogeneity property adds convenient structure without causing any significant restriction.

Definition. An s.i.p. space $V$ has the homogeneity property when the s.i.p. satisfies:

4. $[x, \lambda y]=\bar{\lambda}[x, y]$ for all $x, y \in V$ and all complex $\lambda$.

Adapting the corresponding theorem of $[8$, p. 31$]$ we have

THEOREM 1. Every normed vector space can be represented as an s.i.p. space with the homogeneity property.

Proof. Let $V$ be a normed vector space. By the Hahn-Banach theorem, for each $x \in S$ there exists at least one continuous linear functional, and we choose exactly one, $f_{x} \in S^{*}$ such that $f_{x}(x)=1$. For $\lambda x \in V$ where $x \in S$ and any complex $\lambda$ we choose $f_{\lambda x} \in V^{*}$ such that $f_{\lambda x}=\bar{\lambda} f_{x}$.

Given one such mapping from $V$ into $V^{*}$, (and there exists in general an infinite number of such mappings), it is readily verified that the function

$$
[x, y]=f_{y}(x)
$$

satisfies the properties $1-4$ for an s.i.p.

Throughout this paper it will be assumed that all s.i.p. spaces possess this homogeneity property.

An s.i.p. space has considerable structure when it possesses a continuity property on the right-hand member of the s.i.p.

Definition. A continuous s.i.p. space is an s.i.p. space $C$ where the s.i.p. has the additional property:

5. For every $x, y \in S$

$$
\mathscr{R}\{[y, x+\lambda y]\} \rightarrow \mathscr{R}\{[y, x]\} \text { for all real } \lambda \rightarrow 0 .
$$

The space is a uniformly continuous s.i.p. space when it possesses the property:

5u. The limit in 5 is approached uniformly for all $(x, y) \in S \times S$.

A relation, that we might call an orthogonality relation, can be defined on an s.i.p. space $V$. 
Definition. For $x, y \in V, x$ is normal to $y$ and $y$ is transversal to $x$ if $[y, x]=0$. A vector $x \in V$ is normal to a subspace $N$ and $N$ is transversal to $x$, if $x$ is normal to all vectors $y \in N$.

It follows from properties 1,2 , and 4 that a vector is normal and transversal to every vector in $V$ if and only if it is the null vector.

Since the s.i.p. is not commutative this orthogonality relation is not symmetric, i.e., if $x$ is normal to $y$ then $y$ is not necessarily normal to $x$. However it follows from property 1 that the relation is additive, i.e., if $x$ is normal to $y$ and to $z$ then $x$ is normal to $\lambda y+\mu z$ for all complex $\lambda, \mu$.

For a normed vector space, R. C. James [7] studied the orthogonality relation defined by:

$x$ is orthogonal to $y$ if $\|x+\lambda y\| \geqq\|x\|$ for all scalar $\lambda$.

We show that in a continuous s.i.p. space our s.i.p. orthogonality relation is equivalent to James' norm orthogonality relation.

THEOREM 2. In a continuous s.i.p. space $x$ is normal to $y$ if and only if $\|x+\lambda y\| \geqq\|x\|$ for all complex $\lambda$.

Proof. If $x$ is normal to $y$ then

$$
\begin{aligned}
\|x+\lambda y\|\|x\| & \geqq|[x+\lambda y, x]| \\
& =\left|\|x\|^{2}+\lambda[y, x]\right|=\|x\|^{2},
\end{aligned}
$$

therefore $\|x+\lambda y\| \geqq\|x\|$ for all complex $\lambda$.

Conversely, if $\|x+\lambda y\|-\|x\| \geqq 0$ for all complex $\lambda$, then

$$
\|x+\lambda y\|^{2}-\|x\|\|x+\lambda y\| \geqq 0 .
$$

Therefore $\mathscr{R}\{[x, x+\lambda y]\}+\mathscr{R}\{\lambda[y, x+\lambda y]\}-|[x, x+\lambda y]| \geqq 0$, which implies that $\mathscr{R}\{\lambda[y, x+\lambda y]\} \geqq 0$ for all complex $\lambda$.

Therefore for real $\lambda$,

$$
\begin{aligned}
\mathscr{R}\{[y, x+\lambda y]\} & \geqq 0 \quad \text { for } \lambda \geqq 0, \\
& \leqq 0 \quad \text { for } \lambda \leqq 0 .
\end{aligned}
$$

By the continuity condition 5 , we have for real $\lambda, \mathscr{R}\{[y, x+\lambda y]\} \rightarrow \mathscr{R}\{[y, x]\}$ through positive values for $\lambda \rightarrow 0+$ and through negative values for $\lambda \rightarrow 0-$.

Therefore $\mathscr{R}\{[y, x]\}=0$.

For imaginary $\lambda$, say $\lambda=i \lambda_{1}$ where $\lambda_{1}$ real,

$$
\mathscr{R}\{\lambda[y, x+\lambda y]\}=\lambda_{1} \mathscr{R}\left\{\left[i y, x+\lambda_{1} i y\right]\right\} \geqq 0
$$

and again by the continuity condition $5 \mathscr{R}\{[i y, x]\}=0$, i.e., $\mathscr{I}\{[y, x]\}=0$.

Therefore $[y, x]=0$. 
Definition. A normed vector space is Gâteaux differentiable if for all $x, y \in S$ and real $\lambda$,

$$
\lim _{\lambda \rightarrow 0} \frac{\|x+\lambda y\|-\|x\|}{\lambda} \text { exists. }
$$

A normed vector space is uniformly Fréchet differentiable if this limit is approached uniformly for $(x, y) \in S \times S$.

Now we have as a corollary to Theorem 2 that James' norm orthogonality in a continuous s.i.p. space is additive. But James proves that norm orthogonality is additive if and only if the norm is Gâteaux differentiable [7, p. 274]. Therefore we can conclude that our continuity property 5 is equivalent to Gâteaux differentiability of the norm. However, this can be established directly as follows:

THEOREM 3. An s.i.p. space is a continuous s.i.p. (uniformly continuous s.i.p.) space if and only if the norm is Gâteaux (uniformly Fréchet) differentiable.

Proof. Let $V$ be an s.i.p. space.

For $x, y \in S$ and real $\lambda>0$

(i)

$$
\begin{aligned}
\frac{\|x+\lambda y\|-\|x\|}{\lambda} & \geqq \frac{|[x+\lambda y, x]|-\|x\|^{2}}{\lambda\|x\|} \\
& \geqq \frac{\mathscr{R}\{[x+\lambda y, x]\}-\|x\|^{2}}{\lambda\|x\|} \\
& =\frac{\mathscr{R}\{[y, x]\}}{\|x\|} .
\end{aligned}
$$

But also

$$
\begin{aligned}
\frac{\|x+\lambda y\|-\|x\|}{\lambda} & \leqq \frac{\|x+\lambda y\|^{2}-|[x, x+\lambda y]|}{\lambda\|x+\lambda y\|} \\
& \leqq \frac{|[x, x+\lambda y]|+\lambda \mathscr{R}\{[y, x+\lambda y]\}-|[x, x+\lambda y]|}{\lambda\|x+\lambda y\|} \\
& =\frac{\mathscr{R}\{[y, x+\lambda y]\}}{\|x+\lambda y\|} .
\end{aligned}
$$

Inequalities (i) and (ii) show that the continuity property $5(5 \mathrm{u})$ implies that the norm is Gâteaux (uniformly Fréchet) differentiable, and that the differential is $\mathscr{R}\{[y, x]\} /\|x\|$.

Conversely, suppose the s.i.p. space $V$ has Gâteaux (uniformly Fréchet) differentiable norm. Since

$$
\frac{\|x+\lambda y\|-\|x\|}{\lambda} \quad(\lambda>0) \geqq \frac{\mathscr{R}\{[y, x]\}}{\|x\|} \geqq \frac{\|x+\lambda y\|-\|x\|}{\lambda} \quad(\lambda<0),
$$

therefore

$$
\lim _{\lambda \rightarrow 0} \frac{\|x+\lambda y\|-\|x\|}{\lambda}=\frac{\mathscr{R}\{[y, x]\}}{\|x\|}
$$


For $x, y \in S$ and real $\lambda>0$,

$$
\begin{aligned}
\frac{\|x+\lambda y\|-\|x\|}{\lambda} & =\frac{\mathscr{R}\{[x, x+\lambda y]\}+\lambda \mathscr{R}\{[y, x+\lambda y]\}-\|x\|\|x+\lambda y\|}{\lambda\|x+\lambda y\|} \\
& \geqq \frac{\mathscr{R}\{[y, x]\}}{\|x\|}
\end{aligned}
$$

from (i). I.e.,

$$
\mathscr{R}\{[x, x+\lambda y]\}-\|x\|\|x+\lambda y\|+\lambda\left\{\mathscr{R}\{[y, x+\lambda y]\}-\mathscr{R}\{[y, x]\} \frac{\|x+\lambda y\|}{\|x\|}\right\} \geqq 0 ;
$$

therefore

$$
\liminf _{\lambda \rightarrow 0+}\{\mathscr{R}\{[x, x+\lambda y]\}-\|x\|\|x+\lambda y\|\} \geqq 0 .
$$

Since the norm is differentiable, we have from (iii) that

$$
\liminf _{\lambda \rightarrow 0+}\left\{\frac{\mathscr{R}\{[x, x+\lambda y]\}-\|x\|\|x+\lambda y\|}{\lambda\|x+\lambda y\|}\right\}
$$

is finite and $\geqq 0$. Therefore

$$
\lim _{\lambda \rightarrow 0+} \frac{\|x+\lambda y\|-\|x\|}{\lambda} \geqq \limsup _{\lambda \rightarrow 0+} \frac{\mathscr{R}\{[y, x+\lambda y]\}}{\|x+\lambda y\|} \geqq \frac{\mathscr{R}\{[y, x]\}}{\|x\|}
$$

and from (i) and (ii) $\lim _{\lambda \rightarrow 0+} \mathscr{R}\{[y, x+\lambda y]\} /\|x+\lambda y\|$ exists and is equal to $\mathscr{R}\{[y, x]\} /\|x\|$.

We conclude that $\mathscr{R}\{[y, x+\lambda y]\} \rightarrow \mathscr{R}\{[y, x]\}$ as $\lambda \rightarrow 0$ when the norm is Gâteaux differentiable (and uniformly for $(x, y) \in S \times S$ when the norm is uniformly Fréchet differentiable).

To extend Hilbert space type argument to the theory of the dual of an s.i.p. space we need to impose further structure on the s.i.p. chiefly to guarantee the existence of normals to closed vector subspaces.

Definition. A normed vector space is uniformly convex if given $\varepsilon>0$ there exists a $\delta(\varepsilon)>0$ such that, for $x, y \in S,\|x+y\| / 2 \leqq 1-\delta(\varepsilon)$ when $\|x-y\|>\varepsilon$.

LEMMA 4. In a continuous s.i.p. space which is uniformly convex and complete in its norm there exists a nonzero vector normal to every proper closed vector subspace.

Proof. It is well known that, in a uniformly convex Banach space, given a proper closed vector subspace $N$ and a vector $y \notin N$, then there exists a unique nonzero vector $x_{0} \in N$ such that

$$
\left\|y-x_{0}\right\|=\inf \{\|y-x\| \mid x \in N\}, \quad[12, \text { p. 110]. }
$$

Writing $z_{0} \equiv y-x_{0}$, then

$$
\left\|z_{0}\right\| \leqq\left\|z_{0}+x\right\| \quad \text { for all } x \in N
$$

i.e., $z_{0}$ is normal to $N$. 
Definition. A normed vector space is strictly convex if whenever $\|x\|+\|y\|=$ $\|x+y\|$ where $x, y \neq 0$, then $y=\lambda x$ for some real $\lambda>0$.

It is convenient to characterize strict convexity of the norm in terms of s.i.p. properties. E. Berkson [1, p. 281] states, and it can be proved simply:

LEMMA 5. An s.i.p. space is strictly convex if and only if whenever $[x, y]=\|x\|\|y\|$ where $x, y \neq 0$, then $y=\lambda x$ for some real $\lambda>0$.

It is well known that uniform convexity implies strict convexity.

In Hilbert space the representation theorem for continuous linear functionals sets up a natural correspondence between vectors and continuous linear functionals by means of the inner product. We show that there is a similar representation theorem in a continuous s.i.p. space which is uniformly convex and complete in its norm. An equivalent form of the following theorem has been proved by James [7, p. 288] but we proceed directly by an adaptation of the corresponding Hilbert space theorem using s.i.p. representation.

THEOREM 6. In a continuous s.i.p. space $C$ which is uniformly convex and complete in its norm, to every continuous linear functional $f \in C^{*}$ there exists a unique vector $y \in C$ such that $f(x)=[x, y]$ for all $x \in C$.

Proof. (Existence). If $f(x)=0$ for all $x \in C$, then we choose $y$ as the null vector in $C$.

If $f(x) \neq 0$ for some $x \in C$, then the null space $N$ of $f$ is a proper closed vector subspace of $C$. Hence by Lemma 4, there exists a nonzero vector $y_{0}$ normal to $N$. When $x \in N, f(x)=[x, y]=0$ for $y=\alpha y_{0}$ any complex $\alpha$.

When $x=y_{0}, f(x)=[x, y]=f\left(y_{0}\right)$ for

$$
\left.y=\left(\overline{f\left(y_{0}\right)}\right) /\left\|y_{0}\right\|^{2}\right) y_{0} .
$$

Since each $x \in C$ can be represented in the form $x=z+\lambda y_{0}$ where $z \in N$ and $y_{0}$ is normal to $N$ and $\lambda=f(x) / f\left(y_{0}\right)$, we have for all $x \in C$

$$
f(x)=f\left(z+\lambda y_{0}\right)=f(x)+\lambda f\left(y_{0}\right)=[z, y]+\lambda\left[y_{0}, y\right]=\left[z+\lambda y_{0}, y\right]=[x, y] .
$$

(Uniqueness). Suppose that there exist vectors $y, y^{\prime} \in C, y \neq y^{\prime}$ such that $f(x)=[x, y]=\left[x, y^{\prime}\right]$ for all $x \in C$. Then $[y, y]=\left[y, y^{\prime}\right] \leqq\|y\|\left\|y^{\prime}\right\|$, therefore $\|y\| \leqq\left\|y^{\prime}\right\|$ and hence $\|y\|=\left\|y^{\prime}\right\|$.

From $\|y\|^{2}=\left[y, y^{\prime}\right]$ it follows that $\|y\|\left\|y^{\prime}\right\|=\left[y, y^{\prime}\right]$, and we conclude from Lemma 5 that $y=y^{\prime}$.

We have then, in such a space, that the mapping $x \rightarrow f_{x}$ induced by the s.i.p. is a one-to-one map of $C$ onto $C^{*}$. It is a simple matter to show that this is a norm-preserving mapping.

To make further progress in duality theory we specify that our space be a uniformly continuous s.i.p. space which is uniformly convex and complete in its norm. It is convenient to introduce 
Definition. A uniform s.i.p. space is a uniformly continuous s.i.p. space $M$ where the induced normed vector space is

6. uniformly convex and

7. complete.

A complete duality between a uniform s.i.p. space $M$ and its dual $M^{*}$ is established by

THeOREM 7. For a uniform s.i.p. space $M$, the dual space $M^{*}$ is a uniform s.i.p. space with respect to the s.i.p. defined by $\left[f_{x}, f_{y}\right]=[y, x]$.

Proof. 1. $\left[f_{x}+f_{y}, f_{z}\right]=\left(f_{x}+f_{y}\right)(z)=f_{x}(z)+f_{y}(z)=\left[f_{x}, f_{z}\right]+\left[f_{y}, f_{z}\right]$. From the righthand distributive rule for $M$ we derive a "left-hand distributive rule" for $M^{*}$ of the form:

$$
\left[f_{z}, f_{x}\right]+\left[f_{z}, f_{y}\right]=[x, z]+[y, z]=[x+y, z]=\left[f_{z}, f_{x+y}\right]
$$

Properties 1-4 follow from the corresponding properties of the s.i.p. in $M$ and the definition of the s.i.p. in $M^{*}$.

$5 \mathrm{u}, 6$. It has been proved [11, p. 647] that a Banach space is uniformly Fréchet differentiable if and only if it is uniformly convex. It follows that $M^{*}$ has property $5 \mathrm{u}$ and is uniformly convex.

7. It is well known that the dual of a Banach space is a Banach space.

It is of interest to note the relation between orthogonality in uniform s.i.p. space $M$ and its dual $M^{*}$ : If $x$ is normal to $y$ in $M$ then $f_{x}$ is transversal to $f_{y}$ in $M^{*}$, and vice versa.

Since finite dimensionality implies many uniformity characteristics, a finitedimensional uniform s.i.p. space $M_{n}$ has a simpler defining structure.

THEOREM 8. Every finite-dimensional, strictly convex, continuous s.i.p. space is a uniform s.i.p. space.

Proof. In a continuous s.i.p. space, the function $f(x, y)=\mathscr{R}\{[y, x]\}$ defined on $S \times S$, is continuous in the sense that $\mathscr{R}\{[y, x+\lambda y]\} \rightarrow \mathscr{R}\{[y, x]\}$ for all real $\lambda \rightarrow 0$. But in a finite-dimensional space $S$ is compact and hence $S \times S$ is compact.

Therefore $\mathscr{R}\{[y, x+\lambda y]\} \rightarrow \mathscr{R}\{[y, x]\}$ for all real $\lambda \rightarrow 0$, uniformly for $(x, y) \in S \times S$.

It is well known that every strictly convex finite-dimensional normed vector space is uniformly convex [12, p. 111], and that every finite-dimensional normed vector space is complete.

3. Examples of uniform s.i.p. spaces. Interest in uniform s.i.p. spaces depends largely on the examples of such spaces and on the insight into the nature of the example spaces given by the uniform s.i.p. space structure.

Minkowskian Space. H. Rund's treatment of Minkowskian space [9], [10] suggests that Minkowskian space can be expressed quite naturally as an affine space associated with a real finite-dimensional uniform s.i.p. space. 
A Minkowskian space is an $n$-dimensional affine space with metric $d(x, y)$ $=F(y-x)$ where $F$ is the Minkowskian norm function of the associated vector space where

(i) $F(x)>0$ for $x \neq 0$,

(ii) $F(\lambda x)=|\lambda| F(x)$, for all real $\lambda$,

(iii) $F(x+y) \leqq F(x)+F(y)$, equality holding for $x, y \neq 0$ if and only if $y=\lambda x$ for some real $\lambda>0$.

(iv) $F(x)$ is of class $C^{2}$ in each of its $n$ arguments, the components of vector $x$.

We aim to determine a suitable form for an s.i.p. in the associated vector space and to verify that the associated vector space is a uniform s.i.p. space. We shall use the summation convention.

Euler's theorem on homogeneous functions gives,

$$
F_{x^{i}}(x) x^{i}=F(x)
$$

and

$$
F_{x^{i} x^{4}}(x) x^{i}=0 .
$$

From the identity $\frac{1}{2} F_{x^{\prime} x^{\prime}}^{2^{\prime}}(x)=F_{x^{\prime}}(x) F_{x^{\prime}}(x)+F(x) F_{x^{1} x^{\prime}}(x)$ and using (2) we have

$$
\frac{1}{2} F_{x^{i} x^{\prime}}^{a^{4}}(x) x^{i}=F(x) F_{x^{\prime}}(x)
$$

and

$$
\frac{1}{2} F_{x^{i} x^{3}}^{2}(x) x^{i} x^{j}=F^{2}(x)
$$

We write $g_{i j}(x)=\frac{1}{2} F_{x^{4} x^{3}}^{2}(x)$ and define our s.i.p. for Minkowskian space by

$$
[y, x]=g_{i j}(x) x^{i} y^{j} .
$$

It is easily seen that this s.i.p. satisfies properties $1,2,4$, and 5 . Property 3 is established from the differentiability property (iv) and the convexity property (iii) as follows:

From a known theorem on convex functions, [6, p. 80],

$$
F_{x^{i} x^{3}}(x) y^{i} y^{j} \geqq 0 \text { for all } x, y .
$$

But we have by the second Mean Value Theorem,

$$
F(y)=F(x)+F_{x^{i}}(x)\left(y^{i}-x^{i}\right)+F_{x^{i} x^{j}}(x+\theta\{y-x\})\left(y^{i}-x^{i}\right)\left(y^{j}-x^{j}\right),
$$

where $0<\theta<1$. Using (1) and (5) we deduce that $F(y) \geqq F_{x^{i}}(x) y^{i}$. From (3) we have then that

$$
g_{i j}(x) x^{i} y^{j}=F(x) F_{x^{\prime}}(x) y^{j} \leqq F(x) F(y),
$$

which is the required inequality.

From our general theory we can deduce the form of the linear functionals in Minkowskian space. The linear functional $f_{x}$ corresponding to vector $x$ is given by

$$
\begin{aligned}
f_{x}(z) & =[z, x] \text { for all } z \\
& =g_{i j}(x) x^{i} z^{j},
\end{aligned}
$$


so that the covariant components of vector $x$ are given by

$$
\xi_{i}=g_{i j}(x) x^{j}
$$

This also gives us the form of the mapping $x \rightarrow f_{x}$ of $M$ onto $M^{*}$.

In his development of the theory of Minkowskian spaces Rund adopts, instead of the strict convexity condition (iii), the stronger Legendre condition of the calculus of variations expressed as:

(7) The quadratic form, $g_{i j}(x) y^{i} y^{j}$ is positive definite for all $x$ and $y,[10$, p. 5]. It is known that this condition implies that det $\left|g_{i j}(x)\right|$ is nonzero for all $x,[10, \mathrm{p} .8]$, thus guaranteeing that the inverse map $f_{x} \rightarrow x$, of $M^{*}$ onto $M$ can be determined for any $x$, from the equations (6). However, the uniform s.i.p. space theory informs us that the inverse map $f_{x} \rightarrow x$, of $M^{*}$ onto $M$ is determined under the weaker strict convexity condition (iii).

The Legendre condition (7) is also imposed to provide that the norm of the dual space possesses the same differentiability properties (iv) as the function $F,[10$, p. 23]. However, without this condition the dual is still a uniform s.i.p. space and the principal dual properties of the space are seen to be derived from this uniform s.i.p. space structure.

There are many important Minkowskian spaces which do not obey the Legendre condition, for example, all the $l_{p}^{n}$-spaces where $2<p<\infty,[10, \mathrm{p}$. 17]. The uniform s.i.p. space theory shows how well behaved such spaces are despite this lack.

$\mathscr{L}_{p}$-space $(1<p<\infty)$. The real Banach space $\mathscr{L}_{p}(X, \mathscr{S}, \mu)$ where $1<p<\infty$ can readily be expressed as a uniform s.i.p. space with s.i.p. defined by

$$
[y, x]=\frac{1}{\|x\|_{p}^{p-2}} \int_{X} y|x|^{p-1} \operatorname{sgn} x d \mu
$$

We first consider

$\mathscr{L}_{p}$-space where $2 \leqq p<\infty$. It is obvious that the function satisfies s.i.p. properties $1,2,4$. We proceed to establish

3. Using Hölder's inequality we have

$$
\left.\left|\int_{X} y\right| x\right|^{p-1} \operatorname{sgn} x d \mu \mid \leqq\left(\int_{X}|x|^{p} d \mu\right)^{1 / q}\left(\int_{X}|y|^{p} d \mu\right)^{1 / p},
$$

where $1 / p+1 / q=1$, and the required inequality follows.

5u. Considering $x, y, z \in S$ we have

$$
|[z, x]-[z, y]| \leqq \int_{X}\left|\left(|x|^{p-2} x-|y|^{p-2} y\right) z\right| d \mu .
$$

If for a certain $t \in X$, we assume $x(t)>0$ and $|x(t)|>|y(t)|$, then

$$
\begin{aligned}
0 & \leqq|x(t)|^{p-2} x(t)-|y(t)|^{p-2} y(t) \\
& \leqq(p-1)|x(t)|^{p-2}(x(t)-y(t)), \quad[6, \text { p. 39] }
\end{aligned}
$$


Therefore in general

$$
\left.|| x\right|^{p-2} x-|y|^{p-2} y|\leqq(p-1)| x-y \mid\left(|x|^{p-2}+|y|^{p-2}\right) .
$$

Now by Hölder's inequality, since $p \geqq 2$,

$$
\int_{X}|x|^{p-2}|(x-y) z| d \mu \leqq\left(\int_{X}|(x-y) z|^{p / 2} d \mu\right)^{2 / p} \leqq\left(\int_{X}|x-y|^{p} d \mu\right)^{1 / p} .
$$

Therefore $|[z, x]-[z, y]| \leqq 2(p-1)\|x-y\|_{p}$, which implies that property $5 \mathrm{u}$ holds.

It has been established that such spaces are uniformly convex [3, p. 403].

We now consider

$\mathscr{L}_{p}$-space where $1<p \leqq 2$. The representation theorem for continuous linear functionals on $\mathscr{L}_{p}$-space where $2 \leqq p<\infty$ now follows from the general results obtained for s.i.p. spaces. The continuous linear functional $f_{x}$ corresponding to vector $x \in \mathscr{L}_{p}$ is given by

$$
f_{x}(z)=\frac{1}{\|x\|_{p}^{p-2}} \int_{X} y|x|^{p-1} \operatorname{sgn} x d \mu \text { for all } y .
$$

Further we have that the dual $\mathscr{L}_{p}^{*}$ of an $\mathscr{L}_{p}$-space where $2 \leqq p<\infty$ is itself a uniform s.i.p. space with s.i.p. defined by

$$
\begin{aligned}
{\left[f_{x}, f_{y}\right] } & =\frac{1}{\|x\|_{p}^{p-2}} \int_{X} y|x|^{p-1} \operatorname{sgn} x d \mu \\
& =\frac{1}{\|\eta\|_{q}^{q-2}} \int_{X} \xi|\eta|^{q-1} \operatorname{sgn} \eta d \mu
\end{aligned}
$$

where $1 / p+1 / q=1$, and $\xi=\left(1 /\|x\|_{p}^{p-2}\right)|x|^{p-1} \operatorname{sgn} x$ and $\eta=\left(1 /\|y\|_{p}^{p-2}\right)|y|^{p-1} \operatorname{sgn} y$.

From this s.i.p. for $\mathscr{L}_{p}^{*}$ we deduce that $\mathscr{L}_{p}^{*}$ where $2 \leqq p<\infty$ is $\mathscr{L}_{q}$ where $1 / p+1 / q=1$. We conclude that $\mathscr{L}_{p}$-space where $1<p<\infty$ can be expressed as a uniform s.i.p. space with our defined s.i.p.

\section{BIBLIOGRAPHY}

1. E. Berkson, Some types of Banach spaces, Hermitian operators and Bade functionals, Trans. Amer. Math. Soc. 116 (1965), 376-385.

2. H. Busemann, The foundations of Minkowskian geometry, Comment. Math. Helv. 24 (1950), 156-186.

3. J. A. Clarkson, Uniformly convex spaces, Trans. Amer. Math. Soc. 40 (1936), 396-414.

4. M. M. Day, Normed linear spaces, Springer, Berlin, 1962.

5. M. R. Fortet, Remarques sur les espaces uniformément convexes, Bull. Soc. Math. France 69 (1941), 23-46.

6. G. H. Hardy, J. E. Littlewood and G. Pólya, Inequalities, 2nd ed., Cambridge Univ. Press, Cambridge, 1959.

7. R. C. James, Orthogonality and linear functionals in normed linear spaces, Trans. Amer. Math. Soc. 61 (1947), 265-292. 
8. G. Lumer, Semi-inner-product spaces, Trans. Amer. Math. Soc. 100 (1961), 29-43.

9. H. Rund, Zur Begründung der Differentialgeometrie der Minkowskischen Räume, Arch. Math. 3 (1952), 60-69.

10. - The differential geometry of Finsler space, Springer, Berlin, 1959.

11. V. Smulian, Sur la dérivabilité de la norme dans l'espace de Banach, Dokl. Akad. Nauk SSSR 27 (1940), 643-648.

12. A. Wilansky, Functional analysis, Blaisdell, New York, 1964.

The University of NewCAstle, New South Wales, Australia 